\title{
Optimality and duality for nonsmooth semi-infinite E-convex multi-objective programming with support functions
}

\author{
Tarek Emam ${ }^{1,2, *}$ \\ ${ }^{1}$ Department of Mathematics, Faculty of Science, Jouf University, P.O. Box 2014, Sakaka, Saudi Arabia \\ 2 Department of Mathematics, Faculty of Science, Suez University, P.O. Box 43533, Suez, Egypt
}

Received: 17 September 2019 / Accepted: 29 June 2020

\begin{abstract}
In this paper, we study a nonsmooth semi-infinite multi-objective E-convex programming problem involving support functions. We derive sufficient optimality conditions for the primal problem. We formulate Mond-Weir type dual for the primal problem and establish weak and strong duality theorems under various generalized E-convexity assumptions.
\end{abstract}

Keywords: Nonsmooth semi-infinite multi-objective optimization / generalized e-convexity / duality

\section{Introduction}

Semi-infinite multi-objective programming consider several conflicting objective functions have to be optimized over a feasible set described by infinite number of inequality constraints. Semi-infinite programming problems have occupied the attention of a number of mathematicians due to their applications in many areas such as in engineering, robotics, and transportation problems, see [1]. Optimality conditions and duality results for semiinfinite programming problems have been studied see, [2-10]. Optimality and duality results for semi-infinite multi-objective programming problems that involved differentiable functions were obtained by Caristi et al. [11]. Several kinds of constraints qualifications were defined by Kanzi and Nobakhtian [12] and they obtained necessary and sufficient optimality conditions for nonsmooth semiinfinite multi-objective programming problems. Mishra et al. [13] proved necessary and sufficient optimality conditions for nondifferential semi-infinite programming problems involving square root of quadratic functions, for more details see [14]. Mond and Schechter [15] have constructed symmetric duality of both Wolfe and MondWeir types for nonlinear programming problems where the objective contains the support function. Optimality and duality for a nondifferentiable nonlinear programming problem involving support function have been obtained by Husain et al. [16], see for more details [17-20]. In other hand, convexity and their generalizations play an important role in optimization theory. Youness point of view of convexity is based on the effecting of an operator $\mathrm{E}$ on

\footnotetext{
* e-mail: drtemam@yahoo.com
}

the domain on which functions are defined [21,22]. This kind of convexity is called E-convexity and can be viewed in many fields such as in differential geometry when a manifold is deformed by an operator $\mathrm{E}$. In the field of physical chemistry an E-convexity can be occurred when the binding force $f$ between elements construct a crystal effect by a solution E. In mathematical programming, the notion of E-convexity of functions plays an important role in solving the problem of type composite model problem [23] such as the problem

$$
\begin{aligned}
& \min \|F\| s . t . x \in M, \text { where } f=\|\|, E(x) \\
& =F(x) \text { and }(f \circ E) x=\|F(x)\| .
\end{aligned}
$$

This paper is organized as follows: In Section 2, we mention some definitions and preliminaries. In Section 3, the sufficient optimality conditions for multi-objective semi-infinite E-convex programming problems involving support functions are established. In Section 4, we formulate Mond-Weir type dual for multi-objective semi-infinite E-convex programming problems involving support functions and establish weak, strong and strictconverse duality theorems under generalized E-convexity assumptions.

\section{Definitions and preliminaries}

In this section, we present some definitions and results, which will be needed in this article. Let $R^{n}$ be the n-dimensional Euclidean space and $R_{+}^{n}$ be the nonnegative orthant of $R^{n}$. Let $\langle.,$.$\rangle denotes the Euclidean inner$ product and $\|$. || be Euclidean norm in $R^{n}$. Given a nonempty 
set $D \subseteq R^{n}$, we denote the closure of $\mathrm{D}$ by $\bar{D}$ and convex cone (containing origin) by cone $(D)$. The native polar cone and the strictly negative polar cone are defined respective by

$$
\begin{gathered}
D^{\leq}:=\left\{d \in R^{n} \mid\langle x, d\rangle \leq 0, \forall x \in D\right\}, \\
D^{<}:=\left\{d \in R^{n} \mid\langle x, d\rangle<0, \quad \forall x \in D\right\} .
\end{gathered}
$$

Definition 1 [24] Let $\mathrm{D} \subseteq \mathrm{R}^{\mathrm{n}}$. The contingent cone $T(D, x)$ at $\bar{x} \in \bar{D}$ is defined by

$$
T(D, \bar{x}):=\left\{d \in R^{n} \mid \exists t_{k} \downarrow 0, \exists d_{k} \rightarrow d: \bar{x}+t_{k} d_{k} \in D, \forall k \in N\right\} .
$$

Definition 2 [24] A function $\mathrm{f}: \mathrm{R}^{\mathrm{n}} \rightarrow \mathrm{R}$ is said to be Lipschitz near $\mathrm{x} \in \mathrm{R}^{\mathrm{n}}$, if there exist a positive constant $K$ and a neighborhood $N$ of $x$ such that for any $\mathrm{y}, \mathrm{z} \in \mathrm{N}$, we have

$$
|f(y)-f(z)| \leq K|| y-z|| .
$$

The function $f$ is said to be locally Lipschitz on $R^{n}$ if it is Lipschitz near $x$ for every $x \in R^{n}$.

Definition 3 [24] The Clarke generalized directional derivative of a locally Lipschitz function $\mathrm{f}$ at $x \in \mathrm{R}^{\mathrm{n}}$ in the direction $\mathrm{d} \in \mathrm{R}^{\mathrm{n}}$, denoted by $\mathrm{f}^{\circ}(x, \mathrm{~d})$, is defined as

$$
f^{\circ}(x, d)=\lim _{t \uparrow 0, y \rightarrow x} \frac{\sup (f(y+t d)-f(y))}{t},
$$

where $y \in R^{n}$.

Definition 4 [24] The Clarke generalized subdifferential of $\mathrm{f}$ at $x \in \mathrm{R}^{\mathrm{n}}$ is denoted by $\partial \mathrm{c} \mathrm{f}(x)$, defined as

$$
\partial c f(x)=\left\{\xi \in R^{n}: f^{\circ}(x, d) \geq\langle\xi, d\rangle, \forall d \in R^{n}\right\} .
$$

Definition 5 [21] A set $\mathrm{M} \subseteq \mathrm{R}^{\mathrm{n}}$ is said to be E-convex set with respect to an operator $\mathrm{E}: \mathrm{R}^{\mathrm{n}} \rightarrow \mathrm{R}^{\mathrm{n}}$ if $\lambda \mathrm{E}(x)+$ $(1-\lambda) \mathrm{E}(\mathrm{y}) \in \mathrm{M}$ for each $x, \mathrm{y} \in \mathrm{M}$ and $0 \leq \lambda \leq 1$.

Every $E$-convex set with respect to an operator $E: R^{n} \rightarrow R^{n}$ is a convex set when $E=I$. If $M_{1}$ and $M_{2}$ are $E$-convex sets, then $M_{1} \cap M_{2}$ is $E$-convex set but $M_{1} \cup M_{2}$ is not necessarily $E$-convex set. If $E: R^{n} \rightarrow R^{n}$ is a linear map, and $M_{1}, M_{2} \subseteq R^{n}$ are E-convex sets, then $M_{1}+M_{2}$ is E-convex set.

Example 1 Let $\mathrm{E}: \mathrm{R}^{2} \rightarrow \mathrm{R}^{2}$ be defined as $\mathrm{E}(x, y)=$ $(0, y)$. The set $M=\left\{(x, y) \in R^{2}:(x, y)=\lambda_{1}(0,0)+\right.$ $\left.\lambda_{2}(1,2)+\lambda_{3}(0,3)\right\} \cup\left\{(x, y) \in R^{2}:(x, y)=\lambda_{1}(0,0)+\lambda_{2}\right.$ $\left.\left.(0,-3)+\lambda_{3}(-1,-2)\right\}, \lambda_{i} \geq 0, \sum_{i=1}^{3} \lambda_{i}=1\right\}$ is an E-convex set with respect to the operator $E$.

Definition 6 A locally Lipschitz function $\mathrm{f}: \mathrm{R}^{\mathrm{n}} \rightarrow \mathrm{R}$ is said to be E-convex with respect to an operator $\mathrm{E}: \mathrm{R}^{\mathrm{n}} \rightarrow \mathrm{R}^{\mathrm{n}}$ at $x^{*} \in \mathrm{R}^{\mathrm{n}}$ if

$$
(f \circ E) x-(f \circ E) x^{*} \geq\left\langle\xi, E x-E x^{*}\right\rangle
$$

for each $x \in R^{n}$ and every $\xi \in \partial c f\left(E x^{*}\right)$.

The function $f$ is said to be E-convex near $x^{*} \in R^{n}$ if it is E-convex at each point of neighborhood of $x^{*} \in R^{n}$.
Definition 7 A locally Lipschitz function $\mathrm{f}: \mathrm{R}^{\mathrm{n}} \rightarrow \mathrm{R}$ is said to be strictly E-convex with respect to an operator $\mathrm{E}: \mathrm{R}^{\mathrm{n}} \rightarrow \mathrm{R}^{\mathrm{n}}$ at $x^{*} \in \mathrm{R}^{\mathrm{n}}$ if

$$
(f \circ E) x-(f \circ E) x^{*}>\left\langle\xi, E x-E x^{*}\right\rangle
$$

for each $x \in R^{n}, \quad x \neq x^{*}$ and every $\xi \in \partial c f\left(E x^{*}\right)$.

The function $f$ is said to be strictly E-convex near $x^{*} \in R^{n}$ if it is strictly E-convex at each point of neighborhood of $x^{*} \in R^{n}$.

Proposition 1 [21] If $\mathrm{g}_{i}: \mathrm{R}^{\mathrm{n}} \rightarrow \mathrm{R}, i=1,2, \ldots, \mathrm{m}$ is E-convex with respect to $\mathrm{E}: \mathrm{R}^{\mathrm{n}} \rightarrow \mathrm{R}^{\mathrm{n}}$ then the set $\mathrm{M}=$ $\left\{x \in R^{n}: g_{i}(x) \leq 0, i=1,2, \ldots, m\right\}$ is E-convex set.

Definition 8 A locally Lipschitz function $\mathrm{f}: \mathrm{R}^{\mathrm{n}} \rightarrow \mathrm{R}$ is said to be pseudo E-convex with respect to $\mathrm{E}: \mathrm{R}^{\mathrm{n}} \rightarrow \mathrm{R}^{\mathrm{n}}$ at $x^{*} \in \mathrm{R}^{\mathrm{n}}$ if

$$
\begin{gathered}
\left\langle\xi, E x-E x^{*}\right\rangle \geq 0, \text { for some } \xi \in \partial c f\left(E x^{*}\right) \\
\Rightarrow(f \circ E) x \geq(f \circ E) x^{*},
\end{gathered}
$$

for each $x \in R^{n}$ and every $\xi \in \partial c f\left(E x^{*}\right)$.

Definition 9 A locally Lipschitz function $\mathrm{f}: \mathrm{R}^{\mathrm{n}} \rightarrow \mathrm{R}$ is said to be strictly pseudo E-convex with respect to $\mathrm{E}: \mathrm{R}^{\mathrm{n}} \rightarrow \mathrm{R}^{\mathrm{n}}$ at $x^{*} \in \mathrm{R}^{\mathrm{n}}$ if

$$
\begin{aligned}
& \left\langle\xi, E x-E x^{*}\right\rangle \geq 0, \text { for some } \xi \in \partial c f\left(E x^{*}\right) \Rightarrow(f \circ E) x \\
& \quad>(f \circ E) x^{*},
\end{aligned}
$$

for each $x \in R^{n}, x \neq x^{*}$ and every $\xi \in \partial c f\left(E x^{*}\right)$.

Definition 10 A locally Lipschitz function $\mathrm{f}: \mathrm{R}^{\mathrm{n}} \rightarrow \mathrm{R}$ is said to be quasi E-convex with respect to $\mathrm{E}: \mathrm{R}^{\mathrm{n}} \rightarrow \mathrm{R}^{\mathrm{n}}$ at $x^{*} \in \mathrm{R}^{\mathrm{n}}$ if

$$
(f \circ E) x \leq(f \circ E) x^{*} \Rightarrow\left\langle\xi, E x-E x^{*}\right\rangle \leq 0,
$$

for each $x \in R^{n}$ and every $\xi \in \partial c f\left(E x^{*}\right)$.

The function $f$ is said to be quasi E-convex near $x^{*} \in R^{n}$ if it is quasi E-convex at each point of neighborhood of $x^{*} \in R^{n}$.

Proposition 2 [21] If $\mathrm{g}_{i}: \mathrm{R}^{\mathrm{n}} \rightarrow \mathrm{R}, i=1,2, \ldots, \mathrm{m}$ is quasi $E$-convex with respect to $\mathrm{E}: \mathrm{R}^{\mathrm{n}} \rightarrow \mathrm{R}^{\mathrm{n}}$ then the set $\mathrm{M}=$ $\left\{x \in R^{n}: g_{i}(x) \leq 0, i=1,2, \ldots, \mathrm{m}\right\}$ is E-convex set.

\section{Remark 1}

- Every E-convex function is also quasi E-convex with respect to same $\mathrm{E}: \mathrm{R}^{\mathrm{n}} \rightarrow \mathrm{R}^{\mathrm{n}}$, but not conversely.

- Every E-convex function is also pseudo E-convex with respect to same $\mathrm{E}: \mathrm{R}^{\mathrm{n}} \rightarrow \mathrm{R}^{\mathrm{n}}$, but not conversely.

- Every strictly E-convex function is also strictly pseudo E-convex with respect to same $\mathrm{E}: \mathrm{R}^{\mathrm{n}} \rightarrow \mathrm{R}^{\mathrm{n}}$, but not conversely.

Let $\mathrm{C}$ be a nonempty compact E-convex set in $R^{n}$. The support function $S(. \mid C): R^{n} \rightarrow R \cup\{+\infty\}$ is given by

$$
S(x \mid C)=\max \{\langle x, E z\rangle: z \in C\} .
$$

Example 2 Let $\mathrm{E}: \mathrm{R}^{2} \rightarrow \mathrm{R}^{2}$ be defined as $\mathrm{E}\left(\mathrm{y}_{1}, \mathrm{y}_{2}\right)=(0$, $\left.\mathrm{y}_{2}\right)$. If $C=\left\{\left(y_{1}, y_{2}\right) \in R 2:\left(y_{1}, y_{2}\right)=\lambda_{1}(0,0)+\lambda_{2}(1,2)+\right.$ $\left.\lambda_{3}(0,3)\right\} \cup\left\{\left(y_{1}, y_{2}\right) \in R^{2}:\left(y_{1}, y_{2}\right)=\lambda_{1}(0,0)+\lambda_{2}(0,-3)+\lambda_{3}\right.$ $\left.(-1,-2)\}, \lambda_{i} \geq 0, \sum_{i=1}^{3} \lambda_{i}=1\right\}$, then the support function 
$\mathrm{S}(. / \mathrm{C}): \mathrm{R} \rightarrow \mathrm{R} \cup\{+\infty\}$ is given by

$$
\begin{aligned}
S(x \mid C) & =\max \left\{\left\langle x, E\left(y_{1}, y_{2}\right)\right\rangle:\left(y_{1}, y_{2}\right) \in C\right\} \\
& =\max \left\langle x,\left(0, y_{2}\right)\right\rangle,
\end{aligned}
$$

i.e.

$$
S(x \mid C)=3|x|
$$

The support function, being convex and everywhere finite, has a Clark subdifferential [24], in the sense of convex analysis. Its subdifferential is given by

$$
\partial S(x \mid C)=\{z \in C:\langle x, E z\rangle=S(x \mid C)\} .
$$

In this paper, we consider the following nonsmooth semi-infinite multi-objective E-convex programming problem:

$$
\min f_{j}(x)+S\left(x \mid C_{j}\right), \quad j=1, \ldots, p,
$$

subject to

$$
\begin{gathered}
g_{i}(x) \leq 0, \quad i \in I, \\
x \in R^{n} .
\end{gathered}
$$

where $I$ is an index set which is possibly infinite, $f_{j}(x)$, $j=1, \ldots, p$ and $g_{i}(x), \quad i \in I$ are locally Lipschitz E-convex functions from $R^{n}$ to $R \cup\{+\infty\}$. Let $M$ denote the E-convex feasible set of $(\mathrm{P})$.

$$
M:=\left\{x \in R^{n} \mid g_{i}(x) \leq 0, \forall i \in I\right\}
$$

Let $x^{*} \in M$. We denote $I\left(x^{*}\right)=\left\{i \in I \mid\left(g_{i} \circ E\right) x^{*}=0\right\}$, the index set of active constraints and let

$$
\begin{gathered}
F\left(E x^{*}\right):=\bigcup_{j=1}^{p} \partial_{c}\left(f_{j}\left(E x^{*}\right)+S\left(E x^{*} \mid C_{j}\right)\right) \\
G\left(E x^{*}\right):=\bigcup_{i \in I\left(x^{*}\right)} \partial_{c} g_{i}\left(E x^{*}\right) .
\end{gathered}
$$

The following constraint qualifications are generalization of constraint qualifications from [12] for multiobjective E-convex programming problem with support functions $(\mathrm{P})$.

Definition 11 We say that:

- The Abedie constraint qualification (ACQ) holds at $\tilde{x} \in M$ if $G^{\leq}(\tilde{x}) \subseteq T(M, \tilde{x})$.

- The Basic constraint qualification (BCQ) holds at $\tilde{x} \in M$ if $T \leq(M, \tilde{x}) \subseteq$ cone $(G(x))$.

- The Regular constraint qualification (RCQ) holds at $x \in M$ if $F^{<}(\tilde{x}) \cap G^{\leq}(\tilde{x}) \subseteq T(M, \tilde{x})$.

Definition 12 A feasible point $\mathrm{x}^{*} \in \mathrm{M}$ is said to be weakly efficient solution for $(P)$ if there is no $\mathrm{x} \in \mathrm{M}$ such that

$$
\begin{gathered}
f_{j}(x)+S\left(x \mid C_{j}\right)<f_{j}\left(x^{*}\right)+S\left(x^{*} \mid C_{j}\right), \quad \text { for all } \\
j=1, \ldots, p
\end{gathered}
$$

\section{Optimality conditions}

In this section, we prove the sufficient optimality conditions for considered nonsmooth semi-infinite multiobjective E-convex programming problem $(\mathrm{P})$ as follows:

Theorem 1 (Necessary optimality conditions) Let $\mathrm{E}: \mathrm{R}^{\mathrm{n}} \rightarrow \mathrm{R}^{\mathrm{n}}$ and $x^{*}$ be a feasible solution of $(P)$. Assume that $\mathrm{E} x^{*}$ be a weakly efficient solution of $(P)$ and a suitable constraints qualification from Definition (11) holds at $\mathrm{E}$ $\left(x^{*}\right)$. If cone $\left(G\left(\mathrm{Ex}^{*}\right)\right)$ is closed, then there exist $\tau_{j} \geq 0$, $\mathrm{z}_{j} \in \mathrm{C}_{j}\left(\right.$ for $j=1,2, \ldots, \mathrm{p}$ ) and $\lambda_{i} \geq 0$ (for $\left.i \in \mathrm{I}\left(x^{*}\right)\right)$ with $\lambda_{i} \neq 0$ for finitely many indices $i$, such that

$$
\begin{gathered}
0 \in \sum_{j=1}^{p} \tau_{j}\left[\partial_{c} f_{j}\left(E x^{*}\right)+z_{j}\right]+\sum_{I\left(x^{*}\right)} \lambda_{i} \partial_{c} g_{i}\left(E x^{*}\right), \\
\sum_{j=1}^{p} \tau_{j}=1 \\
\left\langle z_{j}, E x^{*}\right\rangle=S\left(x^{*} \mid C_{j}\right), j=1,2, \ldots, p .
\end{gathered}
$$

Proof: See Theorem 3.4 (ii) of Kanzi and Nobakhtian [12].

Theorem 2 (Sufficient optimality conditions) Let $\mathrm{E}: \mathrm{R}^{\mathrm{n}} \rightarrow \mathrm{R}^{\mathrm{n}}$ and $x^{*}$ be a feasible solution of $(P)$. Assume that there exist $\tau_{j} \geq 0, \mathrm{z}_{j} \in \mathrm{C}_{j}($ for $j=1,2, \ldots, \mathrm{p})$ and $\lambda_{i} \geq 0$ (for $\mathrm{i} \in \mathrm{I}\left(x^{*}\right)$ ) with $\lambda_{i} \neq 0$ for finitely many indices $i$, such that necessary optimality conditions (1)-(3) hold at $x^{*}$. If $\tau_{j}\left(\mathrm{f}_{j}()+.\left\langle\mathrm{z}_{\mathrm{j}},.\right\rangle\right)$ for $j=1,2, \ldots, \mathrm{p}$ are pseudo E-convex at $x^{*}$ and $\lambda_{i} \mathrm{~g}_{i}(),. i \in \mathrm{I}\left(x^{*}\right)$ are quasi $E$-convex at $x^{*}$ with respect to the same $\mathrm{E}$ and $\mathrm{f}_{j}(\mathrm{E} x) \leq \mathrm{f}_{j}(x), j=1, \ldots, \mathrm{p}, \forall x \in \mathrm{M}$. Then, $\mathrm{E} x^{*}$ is a weakly efficient solution for $(P)$.

Proof: Suppose, contrary to the result, that $E x^{*} \in M$, is not a weakly efficient solution for $(\mathrm{P})$. Then, there exists a feasible point $x \in M$ for $(\mathrm{P})$ such that

$$
\begin{gathered}
f_{j}(x)+S\left(x \mid C_{j}\right)<f_{j}\left(E x^{*}\right)+S\left(E x^{*} \mid C_{j}\right), \quad \text { for all } \\
j=1, \ldots, p,
\end{gathered}
$$

but $f_{j}(E x) \leq f_{j}(x)$ and $\tau_{j} \geq 0$, for $j=1,2, \ldots, p$, so we have

$$
\sum_{j=1}^{p} \tau_{j}\left[f_{j}(E x)+S\left(x \mid C_{j}\right)\right]<\sum_{j=1}^{p} \tau_{j}\left[f_{j}\left(E x^{*}\right)+S\left(E x^{*} \mid C_{j}\right)\right] .
$$

Since $\left\langle z_{j}, E x\right\rangle \leq S\left(x \mid C_{j}\right), j=1,2, \ldots, p$ and the assumption $\left\langle z_{j}, E x^{*}\right\rangle=S\left(x^{*} \mid C_{j}\right), j=1,2, \ldots, p$, we have

$$
\sum_{j=1}^{p} \tau_{j}\left[f_{j}(E x)+\left\langle z_{j}, E x\right\rangle\right]<\sum_{j=1}^{p} \tau_{j}\left[f_{j}\left(E x^{*}\right)+\left\langle z_{j}, E x^{*}\right\rangle\right] .
$$

Now, from equation (1), there exist $\xi_{j} \in \partial c f_{j}\left(E x^{*}\right)$ and $\zeta_{i} \in \partial c g_{i}\left(E x^{*}\right)$ such that

$$
\sum_{j=1}^{p} \tau_{j}\left(\xi_{j}+z_{j}\right)+\sum_{I\left(x^{*}\right)} \lambda_{i} \zeta_{i}=0 .
$$


Since $E x$ is a feasible point for $(P)$ where $M$ is E-convex set and $\lambda_{i} g_{i}\left(E x^{*}\right)=0, i \in I\left(x^{*}\right)$, we have

$$
\sum_{I\left(x^{*}\right)} \lambda_{i} g_{i}(E x) \leq \sum_{I\left(x^{*}\right)} \lambda_{i} g_{i}\left(E x^{*}\right)
$$

and from quasi E-convexity of $g_{i}, i \in I\left(x^{*}\right)$, we get

$$
\left\langle E x-E x^{*}, \sum_{I\left(x^{*}\right)} \lambda_{i} \zeta_{i}\right\rangle \leq 0
$$

by using (6), we have

$$
\left\langle E x-E x^{*}, \sum_{j=1}^{p} \tau_{j}\left(\xi_{j}+z_{j}\right)\right\rangle \geq 0 .
$$

Thus, from pseudo E-convexity of $\tau_{j}\left(f_{j}()+.\left\langle z_{j},.\right\rangle\right)$, for $j=1,2, \ldots, p$, we get

$$
\sum_{j=1}^{p} \tau_{j}\left[f_{j}(E x)+\left\langle z_{j}, E x\right\rangle\right] \geq \sum_{j=1}^{p} \tau_{j}\left[f_{j}\left(E x^{*}\right)+\left\langle z_{j}, E x^{*}\right\rangle\right],
$$

which contradicts (5). Thus $E x^{*}$ is a weakly efficient solution for $(\mathrm{P})$.

The following corollary is a direct consequence of Remark 1 and Theorem 2.

Corollary 1 Let $\mathrm{E}: \mathrm{R}^{\mathrm{n}} \rightarrow \mathrm{R}^{\mathrm{n}}$ and $x^{*}$ be a feasible solution of $(P)$. Assume that there exist $\tau_{j} \geq 0, \mathrm{z}_{j} \in \mathrm{C}_{j}$ (for $j=1,2, \ldots, \mathrm{p})$ and $\lambda_{i} \geq O\left(\right.$ for $\left.i \in \mathrm{I}\left(\mathrm{x}^{*}\right)\right)$ with $\lambda_{\mathrm{i}} \neq 0$ for finitely many indices $i$, such that necessary optimality conditions (1)-(3) hold at $x^{*}$. If $\tau_{j}\left(\mathrm{f}_{j}()+.\left\langle\mathrm{z}_{j},.\right\rangle\right)$ for $j=1,2, \ldots, \mathrm{p}$ and $\lambda_{i} \mathrm{~g}_{i}(),. \in \mathrm{I}\left(x^{*}\right)$ are E-convex at $x^{*}$ with respect to the same $\mathrm{E}$ and $\mathrm{f}_{j}(\mathrm{E} x) \leq \mathrm{f}_{j}(x), \mathrm{j}=1, \ldots, p, \forall x \in \mathrm{M}$. Then, $\mathrm{E}^{*}$ is a weakly efficient solution for $(P)$.

Example 3 Let $\mathrm{E}: \mathrm{R}^{2} \rightarrow \mathrm{R}^{2}$ be defined as $E\left(x_{1}, x_{2}\right)=\left(\frac{x_{1}}{2}, x_{2}\right)$ and let $M$ be given by

$$
\begin{aligned}
M & =\left\{\left(x_{1}, x_{2}\right) \in R^{2}: x_{1}+2 x_{2}-6 \leq 0, x_{1}-4 x_{2}\right. \\
& \left.\leq 0, x_{2} \geq 0, x_{1} \geq 0\right\} .
\end{aligned}
$$

Consider the bicriteria $E$-convex programming problem

$$
(P) \min \left(f_{1}(x)+S\left(x \mid C_{1}\right), f_{2}(x)+S\left(x \mid C_{2}\right)\right)
$$

\section{Subjectto $x \in M$,}

where $f_{1}\left(x_{1}, x_{2}\right)=x_{1}^{3}$ and $f_{2}\left(x_{1}, \quad x_{2}\right)=\left(x_{2}-x_{1}\right)^{3}$ and $S\left(x \mid C_{1}\right)=S\left(x \mid C_{2}\right)=\frac{1}{4}\left|x_{2}\right| \quad$ where $\quad x=\left(x_{1}, \quad x_{2}\right)$ for $C_{1}=C_{2}=\left\{\left(0, x_{2}\right):-12 \leq x_{2} \leq 0\right\}$. It is clear that $M$ is $E$ convex with respect to $\mathrm{E}$ and

$E(M)=\left\{\left(x_{1}, x_{2}\right) \in R^{2}: x_{1}+x_{2}-3 \leq 0, x_{1}-2 x_{2} \leq 0, x_{2} \geq 0\right.$, $\left.x_{1} \geq 0\right\}$.

By choosing $\left(g_{1} \circ E\right) x^{*}=x_{1}^{*}-2 x_{2}^{*}$ as the active constraint of $(\mathrm{P})$ then $I\left(x^{*}\right)=1$. It is clear that all defined functions are locally Lipschitz functions at $E x^{*}$ and $\partial f_{1}\left(E x^{*}\right)=\left(12 \alpha^{2}, 0\right), \partial f_{2}\left(E x^{*}\right)=\left(-3 \alpha^{2}, 3 \alpha^{2}\right), \partial g_{1}\left(E x^{*}\right)=$ $(1,-2)$ where $\alpha \in[0,1]$. Since $\tau_{j}\left(f_{j}(x)+\left\langle z_{j}, x\right\rangle\right)$ for $j=1,2$ are pseudo E-convex and $\lambda_{1} g_{1}(x)$ are quasi E-convex at $x^{*}$ with respect to same $E$ and conditions (1)-(3) of theorem (1) holds at $x^{*} \in M$ as there exist $\tau_{1}=\tau_{2}=\lambda=\frac{1}{2}, z_{1}=0$, $z_{2}=-\alpha(9 \alpha+2,3 \alpha+1), \xi_{1}=\left(12 \alpha^{2}, 0\right), \xi_{2}=\left(-3 \alpha^{2}, 3 \alpha^{2}\right)$, $\zeta_{1}=(2 \alpha, \alpha)$, where $\alpha \in[0,1]$ such that

$$
\sum_{j=1}^{2} \tau_{j}\left(\xi_{j}+z_{j}\right)+\sum_{I\left(x^{*}\right)} \lambda_{i} \zeta_{i}=0 .
$$

Then there is no $x \in M$ such that

$$
f_{j}(x)+S\left(x \mid C_{j}\right)<f_{j}\left(E x^{*}\right)+S\left(E x^{*} \mid C_{j}\right), j=1,2,
$$

and hence $E x^{*}=\left(x_{1}^{*}, x_{2}^{*}\right)$ where $x_{1}^{*}=2 x_{2}^{*}$ and $x_{2}^{*} \in[0,1]$ are weakly efficient solutions for $(\mathrm{P})$.

\section{Duality criteria}

Many authors have formulated Mond-Weir type dual and established duality results in various optimization problems with support functions; see $[10,15,17,20,21,25]$ and the references therein. Following the above mentioned works, we formulate Mond-Weir type dual for nonsmooth semiinfinite E-convex programming problem with support function $(\mathrm{P})$ and establish duality theorems.

$\max \left(f_{1}(E y)+\left\langle z_{1}, E y\right\rangle, \ldots, f_{p}(E y)+\left\langle z_{p}, E y\right\rangle\right)$,

subject to

$$
\begin{gathered}
0 \in \sum_{j=1}^{p} \tau_{j}\left[\partial_{c} f_{j}(E y)+z_{j}\right]+\sum_{i \in I} \lambda_{i} \partial_{c} g_{i}(E y), \\
\sum_{i \in I} \lambda_{i} g_{i}(E y) \geq 0 .
\end{gathered}
$$

We now discuss the weak, strong and strict converse duality for $(\mathrm{P})$ and $(\mathrm{D})$.

Theorem 3 (Weak Duality) Let $x$ be feasible for $(P)$ and $\left(\mathrm{y}, \tau, \lambda, \mathrm{z}_{1}, \ldots, \mathrm{z}_{\mathrm{p}}\right)$ be feasible for $(D)$. If $\tau_{j}\left(\mathrm{f}_{j}()+\right.$. $\left.\left\langle\mathrm{z}_{j},.\right\rangle\right)$ for $j=1,2, \ldots, \mathrm{p}$ are pseudo E-convex at $\mathrm{y}$ and $\lambda_{i} \mathrm{~g}_{i}(),. i \in \mathrm{I}$ are quasi $E$-convex at $\mathrm{y}$ with respect to the same $\mathrm{E}$ and $\mathrm{f}_{j}(\mathrm{E} x) \leq \mathrm{f}_{j}(x), j=1, \ldots, p, \forall x \in \mathrm{M}$. Then the following cannot hold:

$$
\begin{gathered}
f_{j}(x)+S\left(x \mid C_{j}\right)<f_{j}(E y)+\left\langle z_{j}, E y\right\rangle, \quad \text { for all } \\
j=1, \ldots, p .
\end{gathered}
$$

Proof: Let $x$ be feasible for $(\mathrm{P})$ and $\left(y, \tau, \lambda, z_{1}, \ldots, z_{p}\right)$ be feasible for (D), then from (8), there exist $\xi_{j} \in \partial c f_{j}(E y)$ and $\zeta_{i} \in \partial c g_{i}(E y)$ such that

$$
\sum_{j=1}^{p} \tau_{j}\left(\xi_{j}+z_{j}\right)+\sum_{i \in I} \lambda_{i} \zeta_{i}=0 .
$$

We proceed to the result of the theorem by contradiction. Assume that

$$
\begin{gathered}
f_{j}(x)+S\left(x \mid C_{j}\right)<f_{j}(E y)+\left\langle z_{j}, E y\right\rangle, \text { forall } \\
j=1, \ldots, p .
\end{gathered}
$$



have

But $f_{j}(E x) \leq f_{j}(x)$ and $\tau_{j} \geq 0$, for $j=1,2, \ldots, p$, so we

$$
\sum_{j=1}^{p} \tau_{j}\left[f_{j}(E x)+S\left(x \mid C_{j}\right)\right]<\sum_{j=1}^{p} \tau_{j}\left[f_{j}(E y)+\left\langle z_{j}, E y\right\rangle\right],
$$

and by using the inequality $\langle z, E x\rangle \leq S(x \mid C)$, we get

$$
\sum_{j=1}^{p} \tau_{j}\left[f_{j}(E x)+\left\langle z_{j}, E x\right\rangle\right]<\sum_{j=1}^{p} \tau_{j}\left[f_{j}(E y)+\left\langle z_{j}, E y\right\rangle\right] .
$$

Now, since Ex is feasible for $(\mathrm{P})$ where $\mathrm{M}$ is E-convex set and $\left(y, \tau, \lambda, z_{1}, \ldots, z_{p}\right)$ is feasible for $(\mathrm{D})$, we have

$$
\sum_{i \in I} \lambda_{i} g_{i}(E x) \leq 0 \leq \sum_{i \in I} \lambda_{i} g_{i}(E y)
$$

and from definition of quasi E-convexity of $g_{i}(x), i \in I$ at $y$, we have

$$
\left\langle E x-E y, \sum_{i \in I} \lambda_{i} \xi_{j}\right\rangle \geq 0,
$$

for each $x \in M$ and every and $\zeta_{i} \in \partial c g_{i}(E x)$. From (10) in (13), we get

$$
\left\langle E x-E y, \sum_{j=1}^{p} \tau_{j}\left(\xi_{j}+z_{j}\right)\right\rangle \geq 0,
$$

for each $x \in M$ and some $\xi_{j} \in \partial c f_{j}(E y)$. Thus, from the definition of pseudo E-convexity of $\tau_{j}\left(f_{j}()+.\left\langle z_{j},.\right\rangle\right)$ for $j=1,2, \ldots, p$, we have

$$
\sum_{j=1}^{p} \tau_{j}\left[f_{j}(E x)+\left\langle z_{j}, E x\right\rangle\right] \geq \sum_{j=1}^{p} \tau_{j}\left[f_{j}(E y)+\left\langle z_{j}, E y\right\rangle\right],
$$

which contradicts (12). Hence,

$$
f_{j}(x)+S\left(x \mid C_{j}\right)<f_{j}(E y)+\left\langle z_{j}, E y\right\rangle, \forall j=1, \ldots, p,
$$

cannot hold.

The following corollary is a direct consequence of Remark 1 and Theorem 3.

Corollary 2 Let $x$ be feasible for $(P)$ and $\left(y, \tau, \lambda, \mathrm{z}_{1}, \ldots\right.$, $\left.z_{p}\right)$ be feasible for $(D)$. If $\tau_{j}\left(\mathrm{f}_{j}()+.\left\langle\mathrm{z}_{j},.\right\rangle\right)$ for $j=1,2, \ldots, p$ are E-convex at $y$ and $\lambda_{i} g_{i}(),. i \in I$ are E-convex at $\mathrm{y}$ with respect to the same $\mathrm{E}$ and $\mathrm{f}_{j}(\mathrm{E} x) \leq \mathrm{f}_{j}(\mathrm{x}), j=1, \ldots, p, \forall x \in \mathrm{M}$. Then the following cannot hold:

$$
f_{j}(x)+S\left(x \mid C_{j}\right)<f_{j}(E y)+\left\langle z_{j}, E y\right\rangle, \forall j=1, \ldots, p .
$$

The following example shows that the generalized B-invexity imposed in the above theorem is essential.

Example 4 [26] We consider the following problem:

$$
(P) \min \left(f_{1}(x)+S\left(x \mid C_{1}\right), f_{2}(x)+S\left(x \mid C_{2}\right)\right)
$$

Subject to

$$
\begin{gathered}
g_{i}(x) \leq 0, i \in I \\
x \in R,
\end{gathered}
$$

where $f_{1}(x)=-2 x, f_{2}(x)=x^{2}, S\left(x \mid C_{1}\right)=S\left(x \mid C_{2}\right)=|x|$ for $C_{1}=C_{2}=[-1,1]$ and $g_{i}(x)=-i|x|$, for $i \in I:=N$. It is clear that the feasible set of $(P)$ is $\mathrm{M}:=\mathrm{R}$ and for $\mathrm{y}=1 \in \mathrm{M}$, $\mathrm{I}(\mathrm{y})=\mathrm{I}$. Let us formulate Mond-Weir dual of $(P)$ as follow:

$$
(D) \max \left\{\left(f_{1} \circ E\right) y+z_{1},\left(f_{2} \circ E\right) y+z_{2}\right\}
$$

Subject to

$$
\begin{gathered}
g_{i}(E y) \leq 0, i \in I \\
0 \in \sum_{j=1}^{2} \tau_{j}\left[\partial f_{j}(E y)+z_{j}\right]+\sum_{i \in I} \lambda_{i} \partial g_{i}(E y), \\
\sum_{i \in I} \lambda_{i} g_{i}(E y) \geq 0,
\end{gathered}
$$

where $y \in R, \tau_{j} \geq 0, \sum_{j=1}^{2} \tau_{j}=1, \lambda_{i} \geq 0$ with $\lambda=\left(\lambda_{\mathrm{i}}\right)_{\mathrm{i} \in \mathrm{I}} \neq 0$ for finitely many indices $i \in \mathrm{N}$ and $\mathrm{z}_{\mathrm{j}} \in \mathrm{C}_{\mathrm{j}}$ for $\mathrm{j}=1$, 2. By choosing $y^{*}=0, \tau_{1}=\tau_{2}=\frac{1}{2}, \lambda=(1,0,0, \ldots), z_{1}=1$, $z_{2}=0$. We have $\left(\mathrm{y}, \tau, \lambda, \mathrm{z}_{1}, \mathrm{z}_{2}\right)$ be feasible for $(D)$.

Note that $\lambda_{i} g_{i}($.$) is not quasi E$-convex at y with respect to $E(y)=\mathrm{y}$ and that $f_{1}(x)+S\left(x \mid C_{1}\right)=-1<f_{1}(E y)+\left\langle z_{1}\right.$, $E y\rangle=0$ holds. This means that pseudo E-convexity and quasi E-convexity assumptions are essential for weak duality.

The following theorem gives strong duality relation between the primal problem $(\mathrm{P})$ and the dual problem (D).

Theorem 4 (Strong Duality) Let $E: R^{n} \rightarrow R^{n}$ and $x^{*}$ be a feasible solution of $(P)$. Assume that $\mathrm{E} x^{*}$ be a weakly efficient solution of $(P)$ and a suitable constraints qualification from Definition (11) holds at $\mathrm{x}^{*}$ and cone $\left(G\left(x^{*}\right)\right)$ is closed. If the pseudo E-convexity and quasi E-convexity assumptions of the weak duality theorem are satisfied, and $f_{j}(E x) \leq f_{j}(x), \mathrm{j}=1, \ldots, p, \forall x \in \mathrm{M}$. Then there exists $\left(\tau, \lambda, \mathrm{z}_{1}, \ldots, \mathrm{z}_{\mathrm{p}}\right)$ such that $\left(x^{*}, \tau, \lambda, \mathrm{z}_{1}, \ldots, \mathrm{z}_{p}\right)$ is a weakly efficient solution for $(D)$ and the respective objective values are equal.

Proof: Since $E x^{*}$ is a weakly efficient solution for $(\mathrm{P})$ at which the suitable constraints qualification holds and cone $\left(G\left(x^{*}\right)\right)$ is closed, from the Kuhn-Tucker necessary conditions, there exists $\left(\tau, \lambda, z_{1}, \ldots, z_{p}\right)$ such that $\left(x^{*}, \tau\right.$, $\left.\lambda, z_{1}, \ldots, z_{p}\right)$ is feasible for $(\mathrm{D})$. hold:

From weak duality theorem (3), the following cannot

$$
f_{j}(x)+S\left(x \mid C_{j}\right)<f_{j}\left(E x^{*}\right)+\left\langle z_{j}, E x^{*}\right\rangle, \text { for } j=1, \ldots, p .
$$

Since $\langle z, E x\rangle \leq S(x \mid C)$, and $f_{j}(E x) \leq f_{j}(x), j=1, \ldots, p$, we have

$$
f_{j}(E x)+\left\langle z_{j}, E x\right\rangle<f_{j}\left(E x^{*}\right)+\left\langle z_{j}, E x^{*}\right\rangle, \text { for } j=1, \ldots, p
$$


cannot hold, and hence $\left(x^{*}, \tau, \lambda, z_{1}, \ldots, z_{p}\right)$ is a weakly efficient solution for $(\mathrm{D})$ and the objective values of $(\mathrm{P})$ and (D) are equal at $x$.

The following corollary is a direct consequence of Remark 1 and Theorem 4.

Corollary 3 Let $E: R^{n} \rightarrow R^{n}$ and $x^{*}$ be a feasible solution of $(P)$. Assume that $\mathrm{E} x^{*}$ be a weakly efficient solution of $(P)$ and a suitable constraints qualification from Definition (11) holds at $x^{*}$ and cone $\left(G\left(x^{*}\right)\right)$ is closed. If the E-convexity assumptions of the weak duality theorem are satisfied, and $f_{j}(E x) \leq f_{j}(x), j=1, \ldots, p, \forall x \in M$. Then there exists $\left(\tau, \lambda, z_{1}, \ldots, z_{p}\right)$ such that $\left(x^{*}, \tau, \lambda, z_{1}, \ldots, z_{p}\right)$ is a weakly efficient solution for $(D)$ and the respective objective values are equal.

The following theorem gives strict converse duality relation between the primal problem $(\mathrm{P})$ and the dual problem (D).

Theorem 5 (Strict converse duality) Let $\mathrm{E} x^{*}$ be a weakly efficient solution for $(P)$ at which a suitable constraints qualification from Definition 11 holds at $x^{*}$ and cone $\left(G\left(x^{*}\right)\right)$ is closed. Let $\tau_{j}\left(f_{j}()+.\left\langle z_{j},.\right\rangle\right)$ for $j=1$, 2, $\ldots, p$ be pseudo E-convex and $\lambda_{i} g_{i}(),. i \in I$ be quasi $E$-convex with respect to the same $\mathrm{E}$. If $\left(\tilde{x}, \tau, \lambda, z_{1}, \ldots, z_{p}\right)$ is a weak efficient solution for $(D)$ and $\tau_{j}\left(f_{j}()+.\left\langle z_{j},.\right\rangle\right)$ for $j=1$, 2, ..., $p$ are strictly pseudo E-convex at $\tilde{x}$, then $\tilde{x}=x^{*}$

Proof: We prove the result of theorem by contradiction. Assume that $\tilde{x} \neq x^{*}$. Then by strong duality Theorem (4) there exists $\left(\tau, \lambda, z_{1}, \ldots, z_{p}\right)$ such that $\left(E x^{*}\right.$, $\left.\tau, \lambda, z_{1}, \ldots, z_{p}\right)$ is a weakly efficient solution for $(\mathrm{P})$ and the inequality

$$
\begin{gathered}
f_{j}(E \tilde{x})+\left\langle z_{j}, E \tilde{x}\right\rangle \leq f_{j}\left(E x^{*}\right)+\left\langle z_{j}, E x^{*}\right\rangle, \text { for } \\
j=1, \ldots, p,
\end{gathered}
$$

cannot be hold. i.e.

$$
\sum_{j=1}^{p} f_{j}\left(E x^{*}\right)+\left\langle z_{j}, E x^{*}\right\rangle<\sum_{j=1}^{p} f_{j}(E \tilde{x})+\left\langle z_{j}, E \tilde{x}\right\rangle .
$$

Now, since $E x^{*}$ is a weakly efficient solution for $(\mathrm{P}), \lambda_{i} \geq 0$ and $\left(\tilde{x}, \tau, \lambda, z_{1}, \ldots, z_{p}\right)$ is a weakly efficient solution for $(\mathrm{D})$, we have

$$
\sum_{i \in I} \lambda_{i} g_{i}\left(E x^{*}\right) \leq \sum_{i \in I} \lambda_{i} g_{i}(E \tilde{x})
$$

and from the definition of quasi E-convexity of $\lambda_{i} g_{i}(),. i \in I$

$$
\left\langle E x^{*}-E \tilde{x}, \sum_{i \in I} \lambda_{i} \zeta_{i}\right\rangle \leq 0
$$

for every $x^{*} \in M$ and every $\zeta_{i} \in \partial c g_{i}(E \tilde{x})$. By substituting from (10) in (15), we get

$$
\left\langle E x^{*}-E \tilde{x}, \sum_{j=1}^{p} \tau_{j}\left(\xi_{j}+z_{j}\right)\right\rangle \geq 0 .
$$

for each $x^{*} \in M$ and some $\xi_{j} \in \partial c f_{j}(E \tilde{x})$. Thus from strict pseudo E-convexity of $\tau_{j}\left(f_{j}()+.\left\langle z_{j},.\right\rangle\right)$ for $j=1,2, \ldots, p$ at $\tilde{x}$, we get

$$
\sum_{j=1}^{p} f_{j}\left(E x^{*}\right)+\left\langle z_{j}, E x^{*}\right\rangle>\sum_{j=1}^{p} f_{j}(E \tilde{x})+\left\langle z_{j}, E \tilde{x}\right\rangle .
$$

which contradicts (1). Therefore, $\tilde{x}=x^{*}$.

\section{Some Applications}

Let us briefly review a few interesting applications. Nonsmooth semi-infinite multi-objective programming problems very naturally lend to a highly disaggregated formulation. Computation of economic equilibria is a very promising area of application for nonsmooth semi-infinite multi-objective programming problems. A paper [27] give example evidence of the solving-power of ACCPM (analytical center cutting plane method) on these reputedly difficult problems. At the end, we would like to mention applications to nonsmooth semi-infinite multiobjective programming problems. In the first application [28], ACCPM (analytical center cutting plane method) is used to solve a Lagrangian relaxation of the capacitate multi-item lot sizing problem with set-up times. A full integration of ACCPM in a column generation, or Lagrangian relaxation, framework, for structured nonsmooth semi-infinite multi-objective programming problems, shows that the reliability and robustness of ACCPM in applications where a non-differentiable problem must be solved repeatedly makes it a very powerful alternative to sub gradient optimization [29].

\section{Conclusions}

This paper investigates the optimality conditions and duality for nonsmooth semi-infinite E-convex multi-objective programming with support functions. The obtained results extended and improved corresponding results of $[26,21]$ to nonsmooth E-convex case. By applying the obtained results, one can study fractional programming, set-valued optimization and variational inequalities and so on. For instance, we can apply the obtained Kuhn-Tucker necessary and sufficient conditions to study the optimality conditions and duality for nonsmooth multiobjective programming problems with generalized E-convexity. We can also apply the Kuhn-Tucker sufficient conditions to consider the solvability of some vector variational inequalities.

\section{References}

1. M.A. Lopez, G. Still, Semi-infinite programming, Eur. J. Oper. Res. 180, 491-518 (2007) 
2. T.D. Chuong, S.D. Kim, Nonsmooth semi-infinite multiobjective optimization problems, J. Optim. Theory Appl. 160, 748-762 (2014)

3. T. Emam, Optimality for E-[0,1] convex Multi-objective Programming, Filomat 31, 529-541 (2017)

4. N. Kanzi, Neccessary optimality conditions for nonsmooth semi-infinite programming problems, J. Global Optim. 49, 713-725 (2011)

5. N. Kanzi, S. Nobakhtian, Optimality conditions for nonsmooth semi-infinite programming, Optimization 53, 717-727 (2008)

6. O.I. Kostyukova, T.V. Tchemisova, Sufficient optimality conditions for convex semi-infinite programming, Optim. Methods Softw. 25, 279-297 (2010)

7. S.K. Mishra, M. Jaiswal, H.A. Thi Le, Duality for nonsmooth semi-infinite programming problems, Optim. Lett. 6, 261-271 (2012)

8. T.Q. Son, J.J. Strodiot, V.H. Nguyen, $\varepsilon$-optimality and $\varepsilon$-Lagrangian duality for a nonconvex programming problem with an infinite number of constraints, J. Glob. Optim. 141 389-409 (2009)

9. T.Q. Son, D.S. Kim, $\varepsilon$-mixed type duality for nonconvex multi-objective programs with an infinite number of constraints, J. Glob. Optim. 57, 447-465 (2013)

10. E.A. Youness, T. Emam, Characterization of efficient solutions of multi-objective optimization problems involving semi-strongly and generalized semi-strongly E-convexity, Acta Math. Sci. 28, 7-16 (2008)

11. G. Caristi, M. Ferrara, A. Stefanescu, Semi-infinite multiobjective programming with generalized invexity, Math. Rep. 12, 217-233 (2010)

12. N. Kanzi, S. Nobakhtian, Optimality conditions for nonsmooth semi-infinite multi-objective programming, Optim. Lett. 8, 1517-1528 (2014)

13. S.K. Mishra, M. Jaiswal, Optimality conditions and duality for nondifferential multi-objective semi-infinite programming, Viet. J. Math. 402\&3, 331-343 (2012)

14. V. Preda, E. Koller, Duality for a nondifferentiable programming problem with a square root term, Rev. Roum. Math. Pures Appl. 45, 873-882 (2000)

15. B. Mond, M. Schechter, Nondifferentiable symmetric duality, Bull. Aust. Math. Soc. 53, 177-188 (1996)
16. I. Husain, Abha, Z. Jabeen, On nonlinear programming with support functions, J. Appl. Math. Comput. 10, 83-99 (2002)

17. T. Antczak, V. Singh, Optimality and duality for minmax fractional programming with support functions under B-(p, r) -Type I assumptions, Math. Comput. Model. 57, 1083-1100 (2013)

18. I. Husain, Z. Jabeen, On fraction programming containing support functions, J. Appl. Math. Comput. 18, 361-376 (2005)

19. D.S. Kim, K.D. Bae, Optimality conditions and duality for a class of nondifferentiable multi-objective programming problems', Taiwan. J. Math. 13, 789-804 (2009)

20. D.S. Kim, H.J. Lee, Optimality conditions and duality in non-smooth multi-objective programs, J. Inequal. Appl. (2010) doi:10.1155/2010/939537

21. E.A. Youness, E-convex sets, E-convex Functions and E-convex programming, J. Optim. Theory Appl. 102 (1999)

22. E.A. Youness, Optimality criteria in E-convex programming, J. Chaos Solit. Fract. 12, 1737-1745 (2001)

23. V. Jeyakumar, X.Q. Yang, Convex composite multi-objective non-smooth programming, J. Math. Program. 59, 325-343 (1993)

24. F.H. Clarke, Optimization and Nonsmooth Analysis (WileyInterscience, New York, 1983)

25. K.D. Bae, D.S. Kim, Optimality and duality theorems in nonsmooth multi-objective optimization, Fixed Point Theory Appl. 42 (2011) doi:10.1186/1687-1812-2011-42

26. S. Yadvendra, S.K. Mishra, K.K. Lai, Optimality and duality for nonsmooth semi-infinite multi-objective programming with support functions, Yugoslav J. Oper. Res. 27, 205-218 (2017)

27. M. Denault, J.L. Goffin, Variational inequalities with quadratic cuts, Gerad Technical Report G-98-69 (1998), 41 Pages.

28. O. du Merle, J.L. Goffin, C. Trouiller, A Lagrangian Relaxation of the capacitate multi-item lot sizing problem solved with an interior point cutting plane algorithm, Logilab Technical Report 97.5, Department of Management Studies, University of Geneva, Switzerland, 1997

29. O. du Merle, P. Hanson, B. Jaumard, N. Maldenovic, An Interior Point Algorithm for Minimum Sum of Squares Clustering, Gerad Technical Report G-97-53. (1997), 28 Pages

Cite this article as: Tarek Emam, Optimality and duality for nonsmooth semi-infinite e-convex multi-objective programming with support functions, Int. J. Simul. Multidisci. Des. Optim. 11, 17 (2020) 